Relations industrielles

Industrial Relations

\title{
La sécurité des revenus face aux changements de structure, Bureau international du travail, Genève, 1969, 165 pp.
}

\section{Jean Boivin}

Volume 24, numéro 4, 1969

URI : https://id.erudit.org/iderudit/028077ar

DOI : https://doi.org/10.7202/028077ar

Aller au sommaire du numéro

Éditeur(s)

Département des relations industrielles de l'Université Laval

ISSN

0034-379X (imprimé)

1703-8138 (numérique)

Découvrir la revue

Citer ce compte rendu

Boivin, J. (1969). Compte rendu de [La sécurité des revenus face aux

changements de structure, Bureau international du travail, Genève, 1969, 165

pp.] Relations industrielles / Industrial Relations, 24(4), 804-805.

https://doi.org/10.7202/028077ar

Tous droits réservés @ C Département des relations industrielles de l'Université Laval, 1969
Ce document est protégé par la loi sur le droit d'auteur. L'utilisation des services d'Érudit (y compris la reproduction) est assujettie à sa politique d'utilisation que vous pouvez consulter en ligne.

https://apropos.erudit.org/fr/usagers/politique-dutilisation/ 


\section{RECENSIONS}

The Origin and Evolution of the I.L.O. and Its Role in the World Community, by David A. Morse, New York State School of Industrial and Labor Relations, Cornell University, Ithaca, New York, 1969, 125 pp.

Ce petit ouvrage contient le texte de trois conférences que le directeur général du B.I.T. a données à l'université Cornell en 1968.

La première est un résumé succinct de l'origine et du développement historique de cete institution pour la période 1919-1948. La seconde montre comment l'O.I.T. a essayé de répondre aux besoins nouveaux suscités par les transformations profondes et rapides qui ont suivi la seconde guerre mondiale jusqu'à nos jours. Enfin la troisième envisage le rôle que doit jouer l'O.I.T. dans la communauté mondiale.

Si la première partie n'apporte rien de bien original pour quiconque a lu d'autres ouvrages consacrés à l'O.I.T., la seconde et la troisième nous font vivre les difficultés et les problèmes qu'a dû affronter cette institution ainsi que les adaptations qui s'imposent dans ses moyens d'action pour qu'elle continue de remplir ses objectifs.

Cet examen critique a d'autant plus de valeur qu'il n'est pas le fruit d'un simple théoricien, mais qu'il est le résultat de l'expérience de celui qui depuis vingt-deux ans a assumé la responsabilité de diriger cet organisme et en connaît tous les aspects.

Cet ouvrage marqué d'un dynamisme sain et réaliste servira sans aucun doute à aider tous ceux qui sont intéressés au développement et à la paix dans le monde.

Gérard DION
La sécurité des revenus face aux changements de structure, Bureau international du travail, Genève, 1969, 165 pp.

Ce livre porte sur quelques-uns des nombreux effets sociaux qui peuvent résulter des changements de structure survenus dans l'économie, notamment, ceux qui concernent la sécurité des revenus.

Le Bureau international du travail a mené une étude parmi les plus importants pays de l'Europe de l'Ouest et de l'Est afin de connaître les différents mécanismes utilisés pour protéger les travailleurs victimes d'un chômage effectif ou potentiel.

Délibérément située dans la perspective des problèmes et des garanties intéressant les travailleurs en cause, cette étude est fondée sur une série de situations concrètes auxquelles donnent lieu les changements de structure. Elle étudie ainsi successivement la garantie de l'emploi et des revenus en cours d'emploi, la réduction des heures supplémentaires et la régulation des ressources, la garantie des revenus en cas de chômage partiel, les mesures de protection et de compensation en cas de licenciement, la garantie des revenus en cas de chômage total, les aides individuelles à la conversion, à la rééducation et au reclassement professionnels, enfin, les problèmes propres à certaines catégories particulières de travailleurs, tels que les jeunes, les femmes, les personnes âgées, les travailleurs agricoles et les immigrants.

L'attention portée à ces divers problèmes dénote une préoccupation commune visant à accorder aux travailleurs, face aux changements de structure, des garanties de revenus mieux adaptées et plus complètes et même, sous une forme plus élaborée, à leur conférer un 
statut de protection qui les prémunisse contre les fluctuations et la perte de leur emploi.

Cette orientation mérite d'être étudiée avec le plus grand intérêt, car elle recèle de nouvelles justifications pour la politique de sécurité sociale, tout en favorisant sa coordination plus étroite avec la politique de plein emploi et la politique économique. Sous l'aspect social, l'avantage essentiel de cette tendance pourrait être de fonder sur une politique active de sécurité sociale des méthodes de protection plus complètes, mieux adaptées et plus efficaces.

\section{Jean BOIVIN}

De la 《politique des revenus 》 à une politique de répartition, par J. C. Koeune, J.L. Kruseman et P. Mandy. Librairie universitaire, Louvain; et Dunod, Paris, 1967, pp. XVI, + 192.

Depuis une vingtaine d'années les politiques de revenus ont fait l'objet de tentative d'applications variées, mais très limitées, sujettes à de nombreuses controverses. Ces politiques étaient trop souvent établies dans le but d'atteindre des objectifs partiels, et ne constituaient d'ailleurs pas toujours le moyen le plus efficace d'atteindre les objectifs visés. C'est précisément cette inadéquation des moyens utilisés par les gouvernements pour atteindre certains objectifs qui amènent les auteurs du présent ouvrage à préciser la nature des politiques de revenus et les objectifs qu'elles étaient censées atteindre. Ils sont aussi amenés à présenter des politiques alternatives plus consistantes avec les objectifs visés. Cet excellent ouvrage se divise en trois parties formant un tout intégré. Au titre premier, les auteurs discutent des questions relatives à la «stabilité monétaire dans la croissance économique ». Le titre II porte sur le salaire et la productivité dans la croissance économique; alors que le titre III traite du plein-emploi dans la croissance économique.

L'objet de la première partie consiste à mettre en évidence le rôle fondamental joué par le système de prix dans l'adaptation de l'offre à la demande des produits finis et des facteurs de production, tout en tenant compte des change- ments structurels découlant de la croissance économique. Mais cette adaptation se fait sous le signe d'un dilemme. Car lorsqu'on se rapproche d'une situation de plein-emploi, l'équilibre ne peut être atteint qu'à travers des changements de prix et de rémunération. Ce qui, dans certaines conditions, conduit à l'inflation par la demande. Ce mode d'ajustement par la hausse des prix n'est pas sans causer des inconvénients majeurs en ce qui concerne, par exemple, le commerce international. Pour éviter ces difficultés, on doit réduire la demande effective par le recours au contrôle monétaire. En d'autres mots quand les tensions inflationnistes apparaissent, on peut pratiquer une politique keynésienne a Contrario. Mais on constate vite que cette politique économique conduit à une situation de sous-emploi. Car, il est établi que la stabilité des prix requiert un niveau de chômage plus ou moins élevé. Ici d'ailleurs, les auteurs se réfèrent en quelque sorte aux relations d'arbitrage entre les prix et le chômage sans pourtant les nommer comme telles.

En résumé donc, la politique keynésienne, efficace en période de dépression, se traduit par le sous-emploi quand on la pratique a Contrario en période de prospérité. Car conçu pour analyser les situations de sous-emploi, le modèle keynésien peut se limiter à l'analyse des ajustements par les quantités et ignorer l'ajustement par la valeur aussi bien que la question de la répartition. Mais comme les Etats ne peuvent systématiquement avoir recours à une politique conduisant au sous-emploi, la politique keynésienne ne peut leur être d'une grande utilité lorsqu'on est en surchauffe. Il fallait donc trouver une politique de freinage des prix qui sauvegarde le plein-emploi. Une politique dont le «moyen d'action principal est le freinage des rémunérations salariales » semble rencontrer cette exigence. C'est donc le souci d'empêcher la spirale des prix sans mettre en danger l'expansion de l'économie qui a amené le développement des politiques de revenus. Le critère que l'on tend à utiliser dans la mise en place de ces politiques, c'est l'indexation des salaires sur la productivité moyenne. Cette politique ne peut qu'introduire de la rigidité et empêche les véritables forces de l'économie de se révéler. En d'autres mots, l'équilibre économique est mouvant; et 